Research Article

\title{
Electrocaloric Response of Ferroelectric Material Applicable as Electrothermal Transducer
}

\author{
Saber Mohammadi, ${ }^{1}$ Akram Khodayari, ${ }^{1}$ and Arash Ahmadi ${ }^{2}$ \\ ${ }^{1}$ Mechanical Department, Engineering Faculty, Razi University, Kermanshah 6714967346, Iran \\ ${ }^{2}$ Electrical Department, Engineering Faculty, Razi University, Kermanshah 6714967346, Iran
}

Correspondence should be addressed to Akram Khodayari; akramkhodayari@yahoo.com

Received 13 May 2013; Accepted 14 August 2013

Academic Editor: Yatendra S. Chaudhary

Copyright ( 2013 Saber Mohammadi et al. This is an open access article distributed under the Creative Commons Attribution License, which permits unrestricted use, distribution, and reproduction in any medium, provided the original work is properly cited.

\begin{abstract}
Electrocaloric response of the PMN-10PT is measured experimentally and compared with the numerical results. Based on the compatibility of the experimental and numerical results, feasibility of using ferroelectric materials as an electrothermal transducer has been investigated. In this study, electrocaloric response of three different ferroelectric capacitors (PMN-10PT, PMN-25PT, and PZN-4.5PT) under an applied periodic electric field have been investigated. Alternative switching of the electrocaloric elements with specific boundary conditions generates a directed heat flux. It can be concluded that each ferroelectric material can be used as a transducer in a special temperature range that in which it has good electrocaloric response.
\end{abstract}

\section{Introduction}

In the recent years, new types of refrigerators using dielectric materials as their working substance have been the centre of attention in several research fields. Electrocaloric effect (ECE) is the physical phenomenon that occurs in ferroelectric materials causing change in their temperature under applied electric field [1-5]. When electric field in the sample changes periodically, heat is released or absorbed due to the electrocaloric effect as well [1]. In other words, the exchanged heat is a function of the applied electric field. Indeed, the pyroelectric and electrocaloric effects may be seen as direct and inverse electrothermal conversion phenomenon. The ECE effect can be used for refrigeration, whereas the pyroelectric effect can be used for temperature/heat sensors/energy harvesting devices [2]. Energy conversion and electrocaloric effect have been actively studied in recent years with the aim of developing effective generators or cooling devices. Some simulation studies have been performed such as those in [611]. Electrocaloric and the pyroelectric effects connect to the temperature dependence of induced polarization. A theoretical description of the thermopolarization effect was presented in [12], where the appearance of a polarization is proportional to the temperature gradient. An opposite effect can be assumed; that is, appearance of a heat flux in the dielectric is proportional to the rate of polarization variations. Further, the relation of the electrocaloric effect to the remnant and induced polarization of a dielectric was studied by Marvan et al. [1]. The induced electric polarization by an external-bias electric field plays a similar role as spontaneous polarization. The electrocaloric effect is coupled with induced polarization by an AC electric field $[13,14]$. Therefore to obtain a solid state cooling device, an effective thermodynamic cycle and the way of removing heat from the cooled object as well as the considerable magnitude of the electrocaloric effect in ferroelectric coolers are very important. In this paper, the electrocaloric characteristics of PMN-10PT ceramic are determined by direct measurements in an electric field with magnitude of $1 \mathrm{kV} / \mathrm{mm}$ at the room temperature and compared with the numerical results. Further, it is shown how these parameters contribute to the heat flux in a sample with selected boundary conditions and consequently the temperature reduction in one edge of the sample. The simulation results for the physical model of the different ferroelectric materials under an applied periodic electric field are presented. 


\section{Thermodynamics of the Electrocaloric Elements}

The thermodynamic equation of a ferroelectric material may be written as

$$
U=W_{e}+W_{m}+Q
$$

where $U, W_{e}, W_{m}$, and $Q$ are the internal, electrical, mechanical, and thermal energies, respectively. Since no stress is applied to the sample, the term of mechanical energy from (1) can be eliminated. The electrical energy is

$$
d W_{e}=E d D
$$

where $E$ is the electric field and $D$ is the electric displacement induction. Since $D$ is a function of $E$ and temperature $T$, we have

$$
d D=\frac{\partial D}{\partial E} d E+\frac{\partial D}{\partial T} d T
$$

From (1), the variations of internal energy are given by

$$
d U=d W_{e}+d Q=\frac{\partial U}{\partial E} d E+\frac{\partial U}{\partial T} d T .
$$

Replacing the expression of the electrical energy in (4) gives the heat variations as follows:

$$
d Q=\left(\frac{\partial U}{\partial E}-E \frac{\partial D}{\partial E}\right) d E+\left(\frac{\partial U}{\partial T}-E \frac{\partial D}{\partial T}\right) d T .
$$

The entropy $S$ is given by

$$
d S=\frac{d Q}{T}=\frac{1}{T}\left(\frac{\partial U}{\partial E}-E \frac{\partial D}{\partial E}\right) d E+\frac{1}{T}\left(\frac{\partial U}{\partial T}-E \frac{\partial D}{\partial T}\right) d T .
$$

It is an exact differential; then

$$
\frac{\partial M}{\partial T}=\frac{\partial N}{\partial E}
$$

where

$$
M=\frac{1}{T}\left(\frac{\partial U}{\partial E}-E \frac{\partial D}{\partial E}\right), \quad N=\frac{1}{T}\left(\frac{\partial U}{\partial T}-E \frac{\partial D}{\partial T}\right) .
$$

Then, we have

$$
\begin{gathered}
-\frac{1}{T^{2}}\left(\frac{\partial U}{\partial E}-E \frac{\partial D}{\partial E}\right)+\frac{1}{T}\left(\frac{\partial^{2} U}{\partial E \partial T}-E \frac{\partial^{2} D}{\partial E \partial T}\right) \\
=\frac{1}{T}\left(\frac{\partial^{2} U}{\partial E \partial T}-E \frac{\partial^{2} D}{\partial E \partial T}-\frac{\partial D}{\partial T}\right) .
\end{gathered}
$$

From this we have

$$
\left(\frac{\partial U}{\partial E}-E \frac{\partial D}{\partial E}\right)=T \frac{\partial D}{\partial T}
$$

By replacing (10) in (5) and assuming that in any case

$$
\frac{\partial U}{\partial T}=c \gg E \frac{\partial D}{\partial T},
$$

where $c$ is the thermal capacitance [15] the expression of $d Q$ is simplified to

$$
d Q=T \frac{\partial D}{\partial T} d E+c d T
$$

The two distinct effects of pyroelectric and electrocaloric come from the writing of the electric displacement as

$$
D=D_{r}+\varepsilon E,
$$

where $D_{r}$ is the remnant electric displacement and $\varepsilon$ is the dielectric permittivity. As for a ferroelectric material $D \approx P$ [15], (13) can be written as

$$
P=P_{r}+\varepsilon E
$$

where $P$ is the polarization and $P_{r}$ is the remnant polarization. Writing the differential of the induction and replacing it in (12) leads to

$$
d Q=T\left(\frac{\partial P_{r}}{\partial T}+E \frac{\partial \varepsilon}{\partial T}\right) d E+c d T .
$$

The term of $T\left(\partial P_{r} / \partial T+E \partial \varepsilon / \partial T\right) d E$ determines the quantity of the heat released (or absorbed) by a thermal electrocaloric source. The first term in the parenthesis is known as the pyroelectric effect (the quantity of $\partial P_{r} / \partial T$ has the meaning of pyroelectric coefficient), whereas the second one is known as the electrocaloric effect (variations of the dielectric permittivity rather than temperature). In the following numerical simulations, the electrocaloric element is simply modeled by

$$
\frac{\partial T}{\partial t}=\frac{k}{\rho c} \frac{\partial^{2} T}{\partial x^{2}}-\frac{T}{\rho c}\left(\frac{\partial P_{r}}{\partial T}+E \frac{\partial \varepsilon}{\partial T}\right) \frac{d E}{d t} \quad 0 \prec x \prec L,
$$

where $\rho$ and $k$ are the mass density and thermal conductivity, respectively.

\section{Experimental and Numerical Procedure}

In this paper, at first the electrocaloric response of PMN10PT material has been measured and calculated both experimentally and numerically to be compared with each other (Figure 2). The sample size is $10 \times 10 \times 1 \mathrm{~mm}^{3}$. Figure 1 (a) shows the experimental implementation of the sample. These results have been measured using a bipolar electric field with amplitude of $1 \mathrm{kV} / \mathrm{mm}$ at the temperature of $25^{\circ} \mathrm{C}$. A rampwave voltage (Figure 2) generated by a HP33210A function generator was amplified by a Trek20/20A amplifier and then applied to the ceramic sample. The temperature of sample was measured by thermocouple. These results are reproduced using numerical simulation as shown in Figure 2. The experimental and numerical results that correspond to the temperature variations at the heat exchanger $(x=l)$ ends of Figure 1 under applied bipolar electric field pulses have been presented. The periodical switching (the applied periodic sequence of pulses to the ferroelectric capacitor) causes a periodic response in the electrocaloric element. It is observed that the experimental and numerical results agree and are 
TABLE 1: Physical properties of the electrocaloric elements [16-19].

\begin{tabular}{lccc}
\hline Material & $\rho$, Mass density $\left(\mathrm{Kg} / \mathrm{m}^{3}\right)$ & $k$, Thermal conductivity W/(mK) & $c$, Heat capacitance $/(\mathrm{kg} \mathrm{K})$ \\
\hline PMN-25PT & 8000 & 0.25 & 312.5 \\
PZN-4.5PT & 8100 & 0.25 & 200 \\
PMN-10PT & 7500 & 0.25 & 350 \\
\hline
\end{tabular}

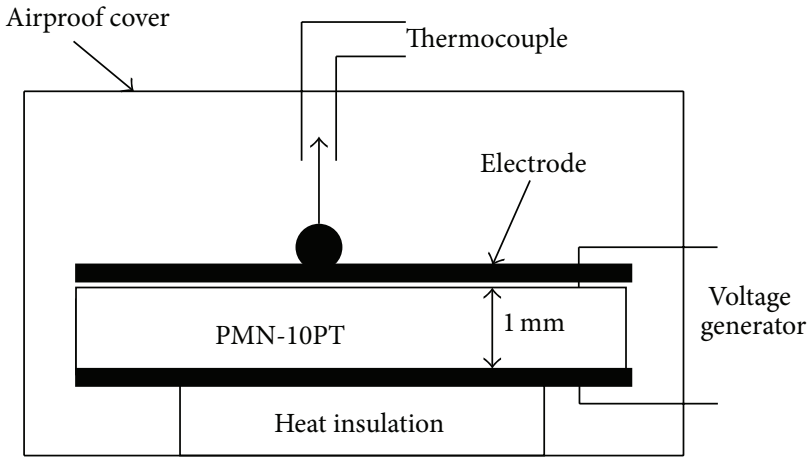

(a)

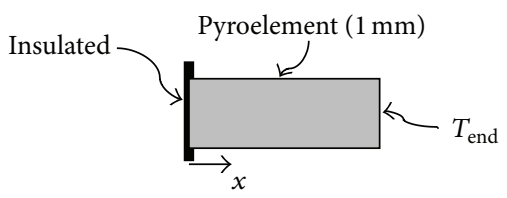

(b)

Figure 1: The sketch map of the EC element temperature measurement and physical model of electrocaloric element.

compatible with each other. Based on this compatibility, it can be concluded that the numerical results can be utilized to estimate the ECE response of other materials. Because of the dielectric susceptibility that determines the value of $d Q(15)$, it is advisable to use the ferroelectric ceramics with the highest value of $\partial \varepsilon / \partial T$ which is important for the electrocaloric heat transformers. Along with the materials revealing big electrocaloric effect, the effective thermodynamic cycle is also important. Then, in this study the PMN-10PT, PMN-25PT and PZN-4.5PT materials have been chosen, whose dielectric constants are rather sensitive to temperature variations. Physical characteristics of these materials are specified in Table 1. In the numerical results, the temperature variations are only examined along the $x$-axis (one-dimensional model). In this case, the temperature distribution $T(x, t)$ along each sample can be found by solving (16), which satisfies the following initial and boundary conditions:

$$
\begin{gathered}
T_{t=0}=T_{\text {end }}{ }^{\circ} \mathrm{C},\left.\quad \frac{\partial T}{\partial x}\right|_{x=0}=0^{\circ} \mathrm{C} / \mathrm{m}, \\
T_{x=l}=T_{\text {end }}{ }^{\circ} \mathrm{C} .
\end{gathered}
$$

One boundary $(x=0)$ is thermally insulated, whereas the other boundary is held at a constant temperature $T_{\text {end }}$ which is the environmental and initial temperature of the sample (Figure 1(b)). Figure 1(b) presents a simple physical

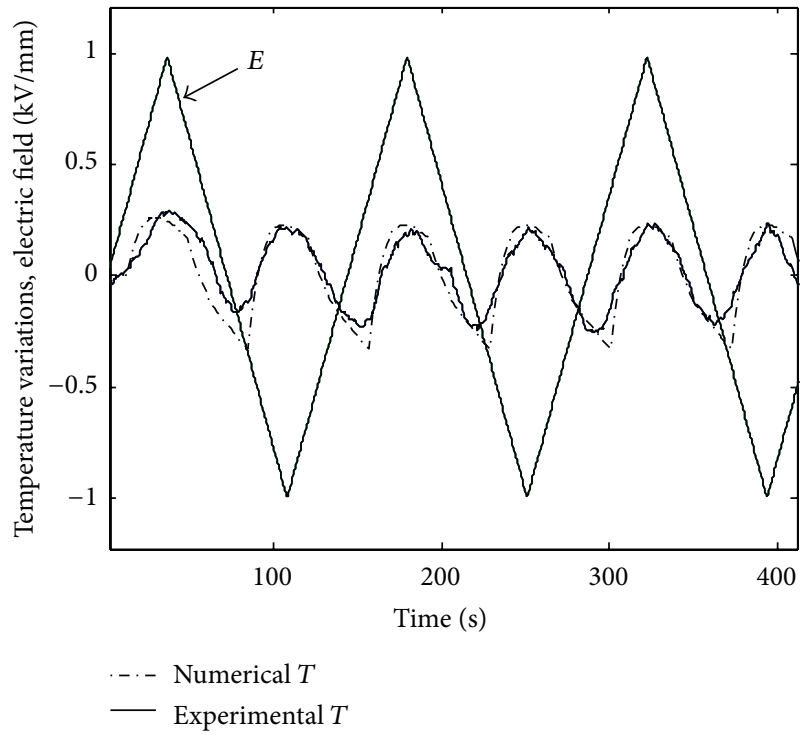

FIGURE 2: Electrocaloric response of PMN-10PT to the applied bipolar electric field.

model, which was used to describe the electrocaloric effect (ECE). It should be mentioned that $T_{\text {end }}$ is $10^{\circ} \mathrm{C}$ for PMN10PT, $170^{\circ} \mathrm{C}$ for PZN-4.5PT, and $120^{\circ} \mathrm{C}$ for PMN-25PT. To have a better electrocaloric response for these materials, these temperatures are selected. In fact, the slop of the Pr-T and $\varepsilon$ - $T$-curves are maximum in these temperatures (Figure 3 ). Therefore, each material is applicable in a specific range of temperature as a transducer or cooling device. In other words, each specific material should be used for special range of temperature (Figure 3). In order to calculate the values of $\partial P_{r} / \partial T$ and $\partial \varepsilon / \partial T$, it is required to determine the change in remnant polarization and dielectric permittivity versus the temperature variations. The values of $\partial P_{r} / \partial T$ and $\partial \varepsilon / \partial T$ for PMN-25PT at $120^{\circ} \mathrm{C}$ have been calculated from the experimental results shown in Figures 3(a) and 3(b) and were determined as follows: $\partial P_{r} / \partial T=-2.68 \times 10^{-3} \mathrm{C} /\left(\mathrm{m}^{2} \cdot \mathrm{K}\right)$ and $\partial \varepsilon / \partial T=1.8 \times 10^{-9} \mathrm{C} /\left(\mathrm{m}^{3} \cdot \mathrm{V} \cdot \mathrm{K}\right)$. The values of $\partial P_{r} / \partial T$ and $\partial \varepsilon / \partial T$ for PZN $-4.5 \mathrm{PT}$ at $170^{\circ} \mathrm{C}$ have been extracted from Figures $3(\mathrm{c})$ and $3(\mathrm{~d})$ as $-0.011 \mathrm{C} /\left(\mathrm{m}^{2} \cdot \mathrm{K}\right)$ and $2.433 \times$ $10^{-8} \mathrm{C} /\left(\mathrm{m}^{3} \cdot \mathrm{V} \cdot \mathrm{K}\right)$, respectively. These values for PMN-10PT at $10^{\circ} \mathrm{C}$ have been extracted from [4] in which $\partial P_{r} / \partial T=$ $-0.012 \mathrm{C} /\left(\mathrm{m}^{2} \cdot \mathrm{K}\right)$ and $\partial \varepsilon / \partial T=3.40725 \times 10^{-9} \mathrm{C} /\left(\mathrm{m}^{3} \cdot \mathrm{V} \cdot \mathrm{K}\right)$. The thermal capacitance $c$ and thermal conductivity $k$ of the materials are assumed to be constant. In this study, the length of the electrocaloric element was $1 \mathrm{~mm}$ (Figure 1(b)). The numerical simulation of the model (16) was performed using the finite-difference algorithm. 


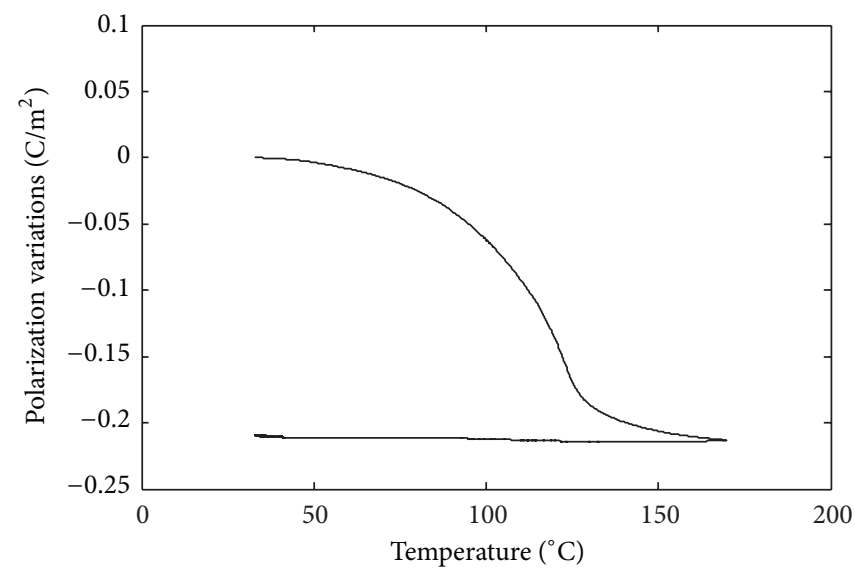

(a) PMN-25PT

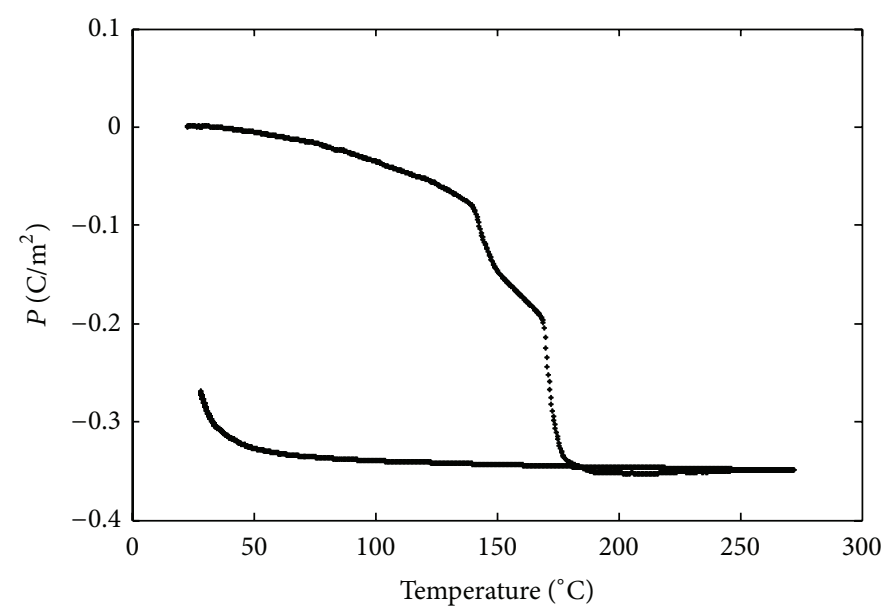

(c) PZN-4.5PT

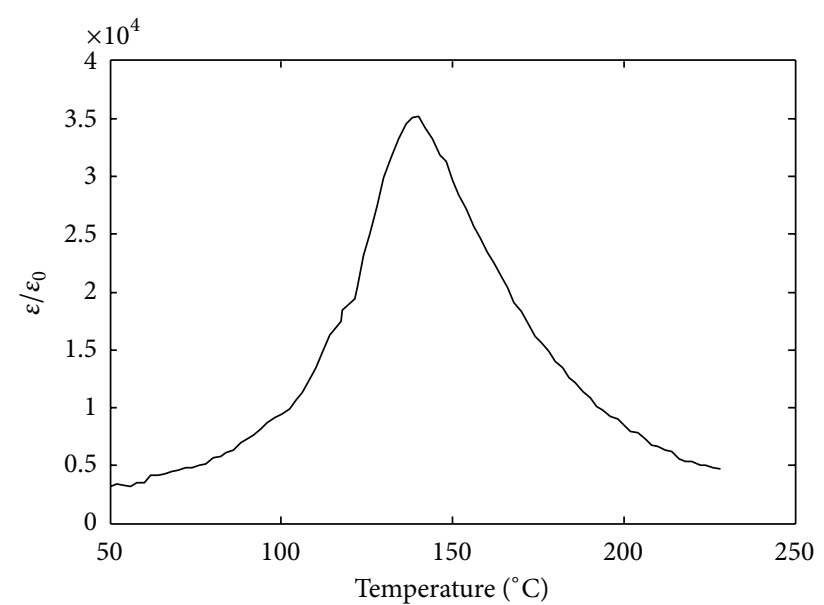

(b) PMN-25PT

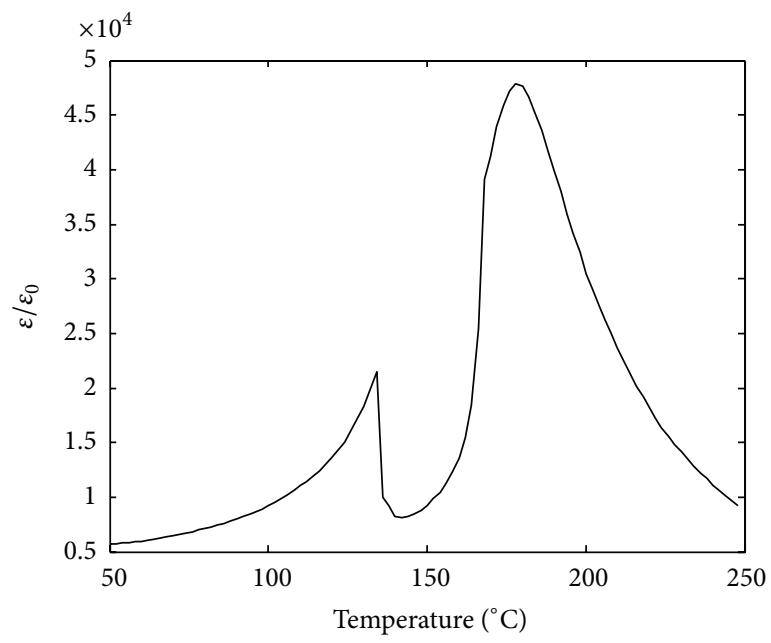

(d) PZN-4.5PT

FIgURE 3: Temperature dependence of the polarization and dielectric constant.

Figure 4 shows the applied electric field pulse to the electrocaloric elements. In this case, we want to reduce temperature by continuous switching of the electric field. Initially, electric field grows relatively fast and quickly switches from zero up to $E_{\max }$ (maximum value of the electric field) in an adiabatic thermal process. The rise time of this switching process is about $10^{-12} \mathrm{~s}$ (interval $t_{0}, t_{1}$ ), which implies that during this short period the electrocaloric element cannot change its temperature immediately. This interval corresponds to the charging of the capacitor and polarization of the ferroelectric. Electric field, which is now equal to $E_{\max }$, is then kept on the EC element during the interval $\left(t_{1}, t_{2}\right)$. Within this interval, the released heat during the charging of the capacitor spreads over the structure and the capacitor remains charged. After the moment $t_{2}$, the electric field is reduced to zero in an adiabatic thermal process $\left(t_{2}, t_{3}\right)$. This interval corresponds to the discharging of the capacitor, which leads to the loosing of temperature. The next pulse is applied immediately to prevent the capacitor from turning back to its initial state, and the cycle is repeated continuously.
The frequency and the amplitude of the applied periodical electric field pulses are $1 \mathrm{~Hz}$ and $1 \mathrm{kV} / \mathrm{mm}$, respectively. The periodical switching (the applied periodic sequence of the pulses to the ferroelectric capacitor) results a periodic cooling (heating) of the electrocaloric elements. This process leads to a redistribution of the temperature field along the structure (i.e., it leads to release or absorption of the thermal energy in the ferroelectric material). After a series of cycles in which the electric field was applied to the model, the temperature distribution between the insulated $(x=0)$ and the heat exchanger $(x=l)$ ends of the EC element has been calculated.

Figure 5 presents the numerical results of the temperature distribution along the sample length of Figure 1(b). After a series of switching cycles (about 80 cycles), the system attains a steady-state temperature regime. The periodic temperature inhomogeneity due to these switching cycles is transferred into the sample which in turn generates a heat flux directed along the $x$-axis of the structure. The heat flux is directed from the insulated end (left side) to the surroundings (right side), which leads to the heat removal or to a decrease 


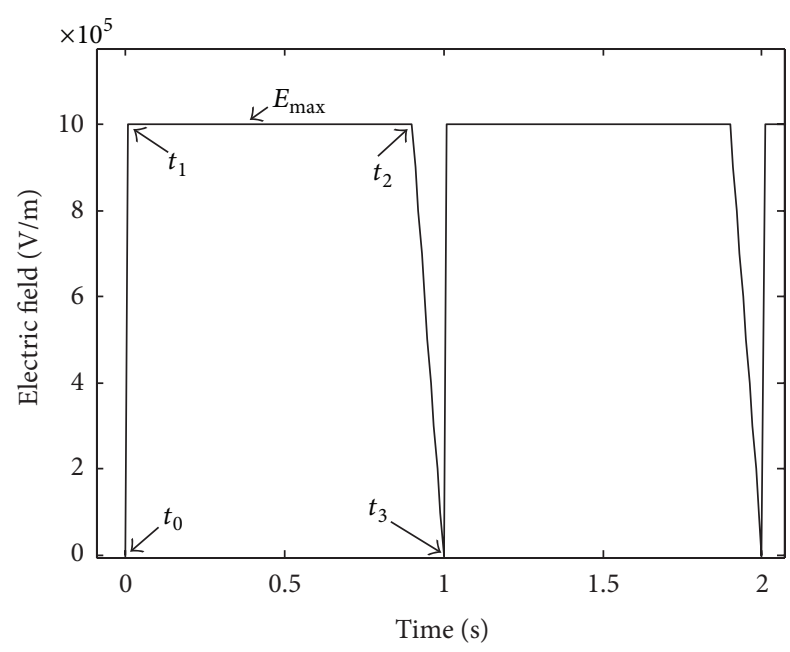

Figure 4: Applied electric pulse to the electrocaloric element.

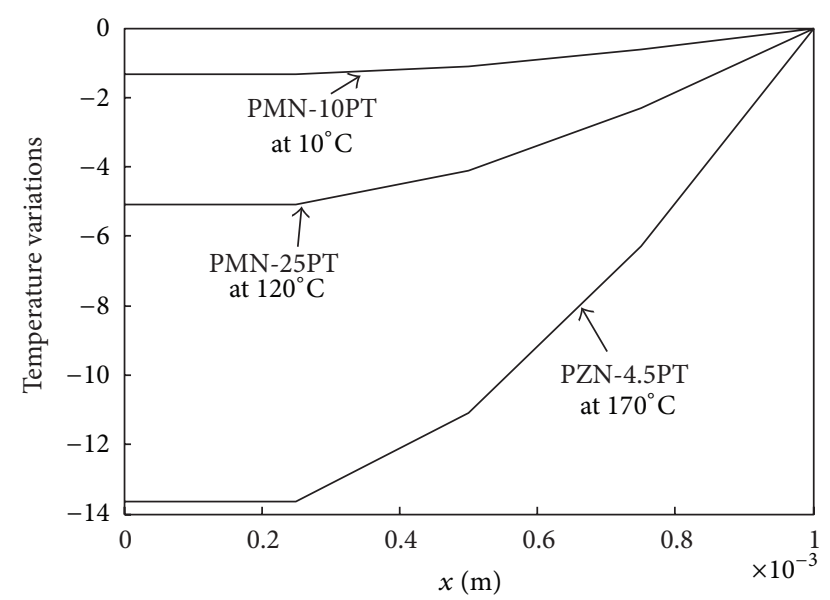

Figure 5: Temperature distribution along the sample length for PMN-10PT, PMN-25PT, and PZN-4.5PT.

in the temperature on the left side. These temperature variations due to the applied periodical electric field to the dielectric material are the bases of the electrocaloric effect. This figure also compares the temperature reduction along the sample length between the three different pyroelectric materials of PMN-10PT, PMN-25PT, and PZN-4.5PT, under their optimum temperatures. Optimum temperature is the one in which the electrocaloric response is maximum. The temperature reduction obtained by $\mathrm{PZN}-4.5 \mathrm{PT}$ is greater than others. This is, to a large extent, caused by $\partial \varepsilon / \partial T$, which is higher in PZN-4.5PT compared with others. The value of the temperature reduction at the insulated end of the sample is about 5 degrees for the PMN-25PT at $120^{\circ} \mathrm{C}, 13.65$ for the $\mathrm{PZN}-4.5 \mathrm{PT}$ at $170^{\circ} \mathrm{C}$, and 1.3 degree for the PMN-10PT at $10^{\circ} \mathrm{C}$.

\section{Conclusions}

This work presents electrocaloric response of the ferroelectric capacitor which can be used as a thermal transducer. It was shown that directed heat flux can be formed in electrocaloric elements. This can be done by periodically switching on the electrocaloric element that creates a temperature gradient which induces a heat flux. Numerical analysis exhibited that a temperature reduction of 5 degree for PMN-25PT at $120^{\circ} \mathrm{C}$, 13.65 degree for $\mathrm{PZN}-4.5 \mathrm{PT}$ at $170^{\circ} \mathrm{C}$, and 1.3 degree for $\mathrm{PMN}$ $10 \mathrm{PT}$ at $10^{\circ} \mathrm{C}$ at the insulated edge of the sample could be achieved. These results indicate that the power of the directed heat flux along the sample strongly depends on the type of the EC material. Also, it is concluded that each ferroelectric material can be used as a transducer in a special temperature range that in which it has good electrocaloric response.

\section{References}

[1] M. Marvan, A. K. Jonscher, and J. Fähnrich, "Electrocaloric effect as a cause of dielectric loss," Journal of the European Ceramic Society, vol. 21, no. 10-11, pp. 1345-1348, 2001.

[2] G. Sebald, L. Seveyrat, D. Guyomar, L. Lebrun, B. Guiffard, and S. Pruvost, "Electrocaloric and pyroelectric properties of $0.75 \mathrm{~Pb}$ $\left(\mathrm{Mg}_{1 / 3} \mathrm{Nb}_{2 / 3}\right) \mathrm{O}_{3}-0.25 \mathrm{PbTiO}_{3}$ single crystals," Journal of Applied Physics, vol. 100, no. 12, Article ID 124112, 2006.

[3] J. Hagberg, A. Uusimäki, and H. Jantunen, "Electrocaloric characteristics in reactive sintered $0.87 \mathrm{~Pb}\left(\mathrm{Mg}_{1 / 3} \mathrm{Nb}_{2 / 3}\right) \mathrm{O}_{3}-0.13$ $\mathrm{PbTiO}_{3}$," Applied Physics Letters, vol. 92, no. 13, Article ID 132909, 2008.

[4] J.-H. Park, K. S. Hong, and S. J. Park, "Frequency dependence of the phase transition in $\mathrm{Pb}\left(\mathrm{Mg}_{1 / 3} \mathrm{Nb}_{2 / 3}\right) \mathrm{O}_{3}-\mathrm{PbTiO}_{3}$ relaxor ferroelectrics," Journal of Applied Physics, vol. 81, no. 4, pp. 20392041, 1997.

[5] S. M. Gupta and D. Viehland, "Influence of non-stoichiometric ordering on the electrostriction coefficient of lead magnesium niobate lead titanate compositions close to the morphotropic phase boundary," Journal of Materials Science, vol. 34, no. 22, pp. 5649-5659, 1999.

[6] A. Khodayari, S. Pruvost, G. Sebald, D. Guyomar, and S. Mohammadi, "Nonlinear pyroelectric energy harvesting from relaxor single crystals," IEEE Transactions on Ultrasonics, Ferroelectrics, and Frequency Control, vol. 56, no. 4, pp. 693-698, 2009.

[7] S. F. Karmanenko, O. V. Pakhomov, A. M. Prudan, A. S. Starkov, and A. V. Es'kov, "Layered ceramic structure based on the electrocaloric elements working as a solid state cooling line," Journal of the European Ceramic Society, vol. 27, no. 8-9, pp. 3109-3112, 2007.

[8] G. Akcay, S. P. Alpay, G. A. Rossetti Jr., and J. F. Scott, "Influence of mechanical boundary conditions on the electrocaloric properties of ferroelectric thin films," Journal of Applied Physics, vol. 103, no. 2, Article ID 024104, 2008.

[9] A. V. Es'kov, S. F. Karmanenko, O. V. Pakhomov, and A. S. Starkov, "Simulation of a solid-state cooler with electrocaloric elements," Physics of the Solid State, vol. 51, no. 8, pp. 1574-1577, 2009.

[10] D. Guyomar, G. Sebald, E. Lefeuvre, and A. Khodayari, "Toward heat energy harvesting using pyroelectric material," Journal of Intelligent Material Systems and Structures, vol. 20, no. 3, pp. 265-271, 2009.

[11] S. Liu and Y. Li, "Research on the electrocaloric effect of PMN/PT solid solution for ferroelectrics MEMS microcooler," Materials Science and Engineering B, vol. 113, no. 1, pp. 46-49, 2004. 
[12] A. K. Tagantsev, "Pyroelectric, piezoelectric, flexoelectric, and thermal polarization effects in ionic crystals," Journal Soviet Physics Uspekhi, vol. 30, no. 7, pp. 588-603, 1987.

[13] M. Marvan, "Influence of electrocaloric effect upon the dynamic susceptibility of multi-domain ferroelectric materials," Czechoslovak Journal of Physics, vol. 19, no. 4, pp. 482-487, 1969.

[14] W. N. Lawless, "Specific heat and electrocaloric properties of $\mathrm{KTaO}_{3}$ at low temperatures," Physical Review B, vol. 16, no. 1, pp. 433-439, 1977.

[15] D. Guyomar, G. Sebald, B. Guiffard, and L. Seveyrat, "Ferroelectric electrocaloric conversion in $0.75\left(\mathrm{PbMg}_{1 / 3} \mathrm{Nb}_{2 / 3} \mathrm{O}_{3}\right)$ $-0.25\left(\mathrm{PbTiO}_{3}\right)$ ceramics," Journal of Physics D, vol. 39, no. 20, article 29, pp. 4491-4496, 2006.

[16] S. Mohammadi and A. Khodayari, "Pyroelectric energy harvesting: with thermodynamic-based cycles," Journal of Smart Materials Research, vol. 2012, Article ID 160956, 5 pages, 2012.

[17] A. Khodayari and S. Mohammadi, "Solid-state cooling line based on the electrocaloric effect," IEEE Transactions on Ultrasonics, Ferroelectrics, and Frequency Control, vol. 58, no. 3, pp. 503-508, 2011.

[18] G. Sebald, S. Pruvost, and D. Guyomar, "Energy harvesting based on Ericsson pyroelectric cycles in a relaxor ferroelectric ceramic," Smart Materials and Structures, vol. 17, no. 1, Article ID 015012, 2008.

[19] G. Sebald, E. Lefeuvre, and D. Guyomar, "Pyroelectric energy conversion: optimization principles," IEEE Transactions on Ultrasonics, Ferroelectrics, and Frequency Control, vol. 55, no. 3, pp. 538-551, 2008. 

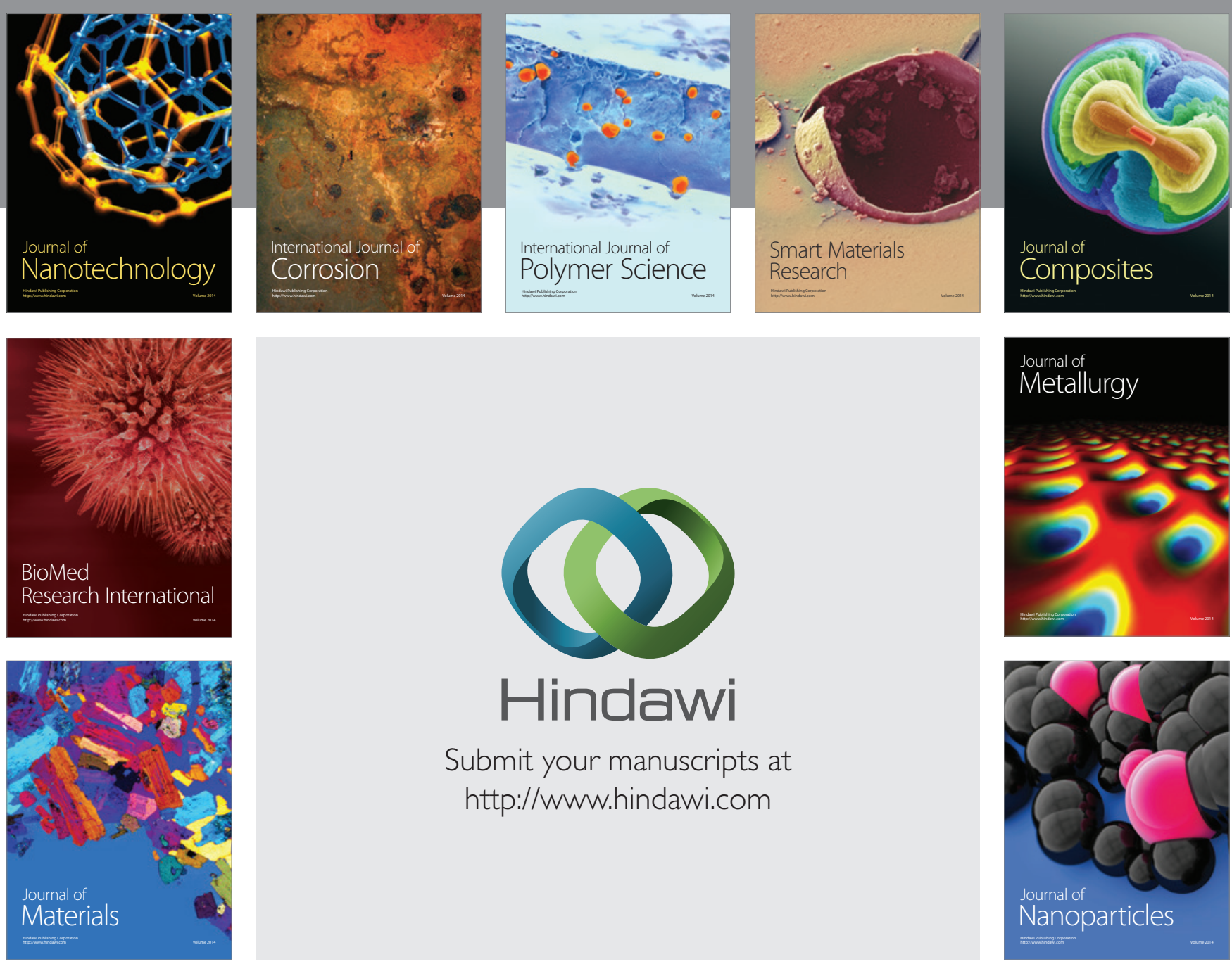

Submit your manuscripts at http://www.hindawi.com
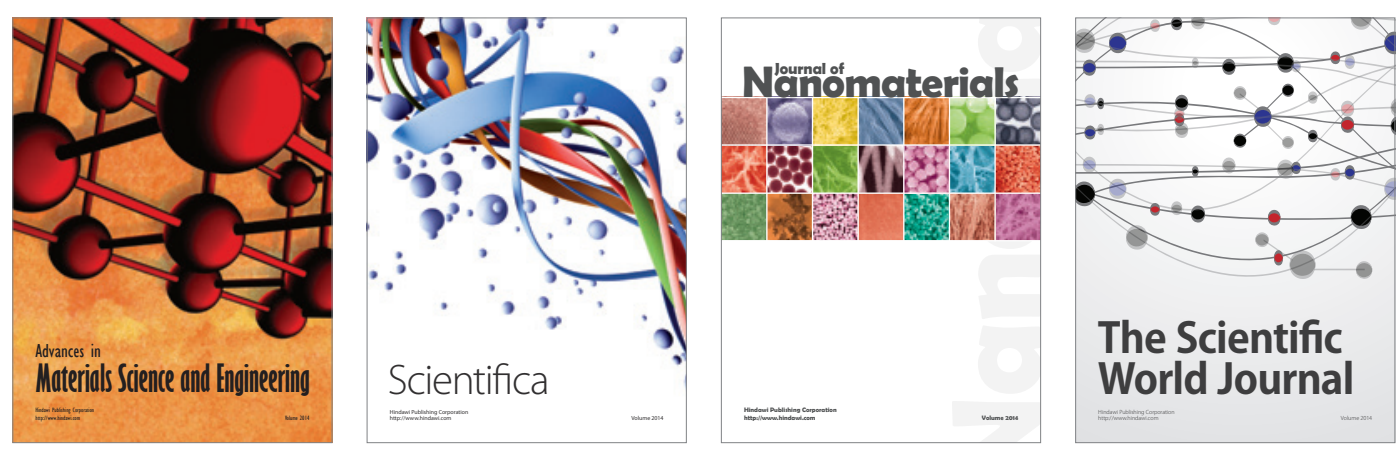

\section{The Scientific World Journal}
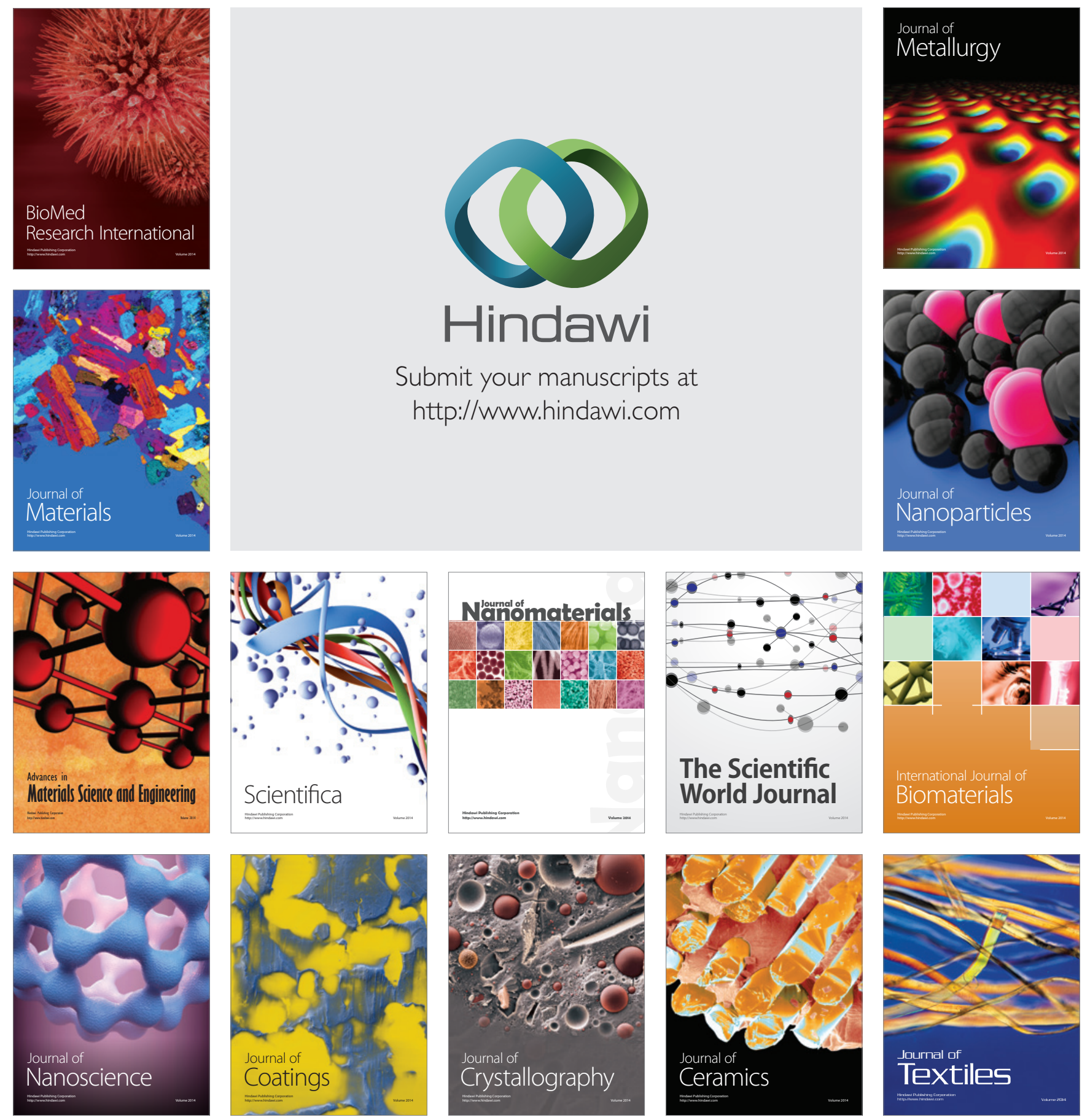